\title{
Stability of columnar order in assemblies of hard rectangles or squares
}

\author{
Trisha Nath ${ }^{1}$, Deepak Dhar ${ }^{2}$ and R. Rajesh ${ }^{1}$ \\ The Institute of Mathematical Sciences - C.I.T. Campus, Taramani, Chennai 600113, India \\ Department of Theoretical Physics, Tata Institute of Fundamental Research, Homi Bhabha Road, Mumbai 400005, \\ India \\ PACS 05.50.+q - Lattice theory and statistics \\ PACS 64.60. De - Statistical mechanics of model systems \\ PACS $64.60 . \mathrm{Bd}$ - General theory of phase transitions

\begin{abstract}
A system of $2 \times d$ hard rectangles on square lattice is known to show four different phases for $d \geq 14$. As the covered area fraction $\rho$ is increased from 0 to 1 , the system goes from low-density disordered phase, to orientationally-ordered nematic phase, to a columnar phase with orientational order and also broken translational invariance, to a high density phase in which orientational order is lost. For large d, the threshold density for the first transition $\rho_{1}^{*}$ tends to 0 , and the critical density for the third transition $\rho_{3}^{*}$ tends to 1 . Interestingly, simulations have shown that the critical density for the second transition $\rho_{2}^{*}$ tends to a non-trivial finite value $\approx 0.73$, as $d \rightarrow \infty$, and $\rho_{2}^{*} \approx 0.93$ for $d=2$. We provide a theoretical explanation of this interesting result. We develop an approximation scheme to calculate the surface tension between two differently ordered columnar phases. The density at which the surface tension vanishes gives an estimate $\rho_{2}^{*}=0.746$, for $d \rightarrow \infty$, and $\rho_{2}^{*}=0.923$ for $d=2$. For all values of $d$, these estimates are in good
\end{abstract} \\ agreement with Monte Carlo data.
}

Introduction. - Models of particles with only excluded volume interaction provide very simple theoretical models of phase transitions. In these models, density is $\checkmark$ the only control parameter, and the phase transitions are entropy-driven, and geometrical in nature. These models serve as good first order models for understanding entropydriven transitions in more complex systems. For instance, the liquid-solid melting transition in real monoatomic or - colloidal solids 1 may be modeled by the hard sphere system that exhibits a discontinuous transition from a fluid phase to a crystalline phase with increasing density [2,3]. Likewise, the qualitative features of isotropic liquid, nematic, smectic and solid phases observed in liquid crystals can be seen in the much simpler models of hard rods or spherocylinders [4 8]. Similarly, transitions in adsorbed monolayers ( $\mathrm{Cl}$ on $\mathrm{Ag}$ ) may be modeled using hard squares on lattices [9]. The different shapes of particles that have been studied include dimers [10 12], trimers [13, hexagons [14, triangles [15], squares [16 21], discs 22, 23, tetrominoes 24, rods 25, 27, and rectangles [28 30]. Despite sustained interest in hard-core models, exact solutions exist only for hard hexagons on the triangular lattice 14 and a special limiting case of thin long cylinders in three dimensional continuum [5].

In this context, the problem of hard $2 \times d$ rectangles on a square lattice is specially interesting. Recently, we showed that a system of $2 \times d$ hard rectangles for $d \geq 14$ shows four distinct phases 28]. The low-density phase is a disordered gas-like phase. On increasing density beyond a critical value $\rho_{1}^{*}(d)$, the system enters a nematic phase, with a larger fraction of rectangles having the same orientation (horizontal, or vertical), but with no breaking of translational invariance. On increasing the density further, beyond a density $\rho_{2}^{*}(d)$, the system enters a columnar phase, where translational symmetry is broken in a direction perpendicular to the orientational order: the system develops a layered structure of period 2, with preferential occupation of particles in either odd, or even rows, when the orientational ordering is horizontal. Finally, beyond a density $\rho_{3}^{*}(d)$, orientational order is lost for all $d$. However, if $d$ is even, sublattice order is present [28, 31, 32.

There has been a lot of interest in the study of columnar, or stripe order in recent years 33. It is encountered in many different settings, such as liquid crys- 
tals 4, frustrated spin systems, both classical 34,35, and quantum 36, 37, and hard core lattice gas models [16, 18, 23, 28, 38. However, our understanding of columnar ordering is still not very satisfactory. For instance, while there exists rigorous proof for the existence of the solid-like sublattice 3941 and nematic phases 42, there is no corresponding result for columnar order.

The prototypical model of columnar order is the classical $2 \times 2$ hard-square lattice gas, which has been studied for a long time [16, 18, 22, 38, 43, 47]. Approximation schemes like different versions of mean-field theory, cluster variational methods, and density functional theory, do not capture the correlations in the columnar phase well, and the estimates of the critical activity $z_{c}(2)$ are off by a factor of 5 from the Monte Carlo results (also see Discussion). In this paper, based on two different approximate calculations of interfacial tension, we obtain $z_{c}(2)=52.49$ and 54.87, and $\rho_{c}(2)=0.923$ and 0.932 for the critical density, to be compared with $z_{c}(2) \approx 97.5$, and $\rho_{c}(2) \approx 0.932$ from Monte Carlo simulations [22,44,45].

We study the transition between the nematic and columnar phases of $2 \times d$ rectangles on a square lattice, under the simplifying restriction that all rectangles are fully aligned (all horizontal). For large $d$, for most densities in the nematic/columnar range, this approximation is very good (for $d=18$ and $\rho=0.75$, the deviation from perfect orientational order is $\approx 0.15 \%$ ). Whenever a nematic phase exists (only when $d \geq 14$ ), the critical threshold for the nematic-columnar transition with all rectangles oriented in one direction was shown numerically to not change much if they are allowed to have arbitrary orientations [48]. Also, it is not an approximation when $d=2$, as there is no distinction between horizontal and vertical squares. However, restricting to only horizontal rectangles has the important consequence that we obtain a phase transition for all $d \geq 2$, while if we allow rectangles of both orientations, then systems with odd $d$ and $d \leq 9$ have no phase transitions 32 .

For large $d$, the estimates from Monte Carlo simulations for the the critical parameters of the nematic-columnar transition are $z_{c}(d) \approx 71.0 d^{-1}+O\left(d^{-2}\right)$ and $\rho_{c}(d) \approx 0.73+$ $0.46 d^{-1}+O\left(d^{-2}\right)$ [31. As in the case of hard squares, standard approximation techniques give poor theoretical estimates. Estimates from the singular high-density expansion in powers of $z^{-1 / d}$ give $z_{c}(d)=5.56 d^{-1}+O\left(d^{-2}\right)$ and $\rho_{c}(d)=0.357+0.73 d^{-1}+O\left(d^{-2}\right)$ 48, whereas a Bethe approximation gives $\rho_{c}(d)=0.59+0.29 d^{-1}+O\left(d^{-2}\right)$ [28].

We estimate the interfacial tension between two phases with different columnar order, and setting this to zero, we obtain a condition for the limit of stability of the columnar ordered phase. We have done our calculation in two different approximations. In the first, we work out the highdensity expansion for the interfacial tension as a perturbation series in the defect density, and estimate the critical point by truncating this expansion at first order. The formal power series in powers of defect density is a singular perturbation series in the activity $z$. At the zeroth order in defect density, it is straightforward to determine the partition function per site in the bulk, and the interface is a partially directed self-avoiding walk (PDSAW). Summing over the different configurations of the walk gives us the zeroth order estimates of $z_{c}(d)$ and $\rho_{c}(d)$. We then calculate the first order correction to this result due to the presence of defects in the bulk phases. In the second method, we ignore defects in the bulk, but take into account a subset of configurations of the interface with overhangs to improve the estimate of the interfacial tension. Both these approximations give values [see Eqs. (18), (19), (22) and [23)] that are quite close to the Monte Carlo estimates, and are a significant improvement over earlier methods.

Model and methodology. - Consider hard rectangles of size $2 \times d(d \geq 2)$, whose long edges are aligned along the horizontal $x$-direction, on a square lattice of size $N \times M$, interacting through only excluded volume interactions (two rectangles may not overlap). An activity $z$ is associated with each rectangle. The system is disordered at low densities and shows columnar order at high densities [48. We call the phase odd (even) in which the majority of heads (bottom left corner) of the rectangles are in odd (even) rows.

The critical density for the nematic-columnar transition is finite because, for large $d$, we can rescale the $\mathrm{x}$-axis by a factor $d$, and the problem becomes equivalent to a model of $1 \times 1$ oriented hard squares in a space where $x$ is continuous, but $y$ is discrete. Two squares cannot overlap or have a common boundary. In this continuum problem, the space between two lines $y=n$ and $y=n+1$ is like a continuum hard-core gas 49,50 , and the transition would be expected to occur at a finite density. The fractional area covered is unaffected by rescaling of the x-axis. The activity $z_{\text {cont }}$ in this continuum model is related to the lattice activity $z$ by $z_{\text {cont }}=d z$.

We estimate the critical activity $z_{c}(d)$ and the critical density $\rho_{c}(d)$ at the nematic-columnar transition by calculating within an approximation scheme, the interfacial tension $\sigma(z)$ between the even and odd phases. Estimating interfacial tension has been useful in determining the phase diagrams of different lattice models. Examples include Ising [51,52, and Potts models 53.

To compute $\sigma(z)$, we impose an interface by fixing the rectangles at the left (right) boundary to be even (odd). A typical configuration seen in a simulation of $2 \times 4$ rectangles is shown in Fig 1(a). We note that there may be some empty space between the odd and even rectangles. To define an unique position of the interface for any allowed configuration of rectangles, we adopt the convention that the boundary between the left (even) and right (odd) phase is placed as far left as possible. With this convention, there is a well-defined interface with the bulk phases having very few defects (rectangles of the wrong type which when removed results in a fully ordered columnar phase).

Let $\Omega_{e o}\left(z, z_{D} \mid I\right)$ be the partition function with a given 
(a)

(b)
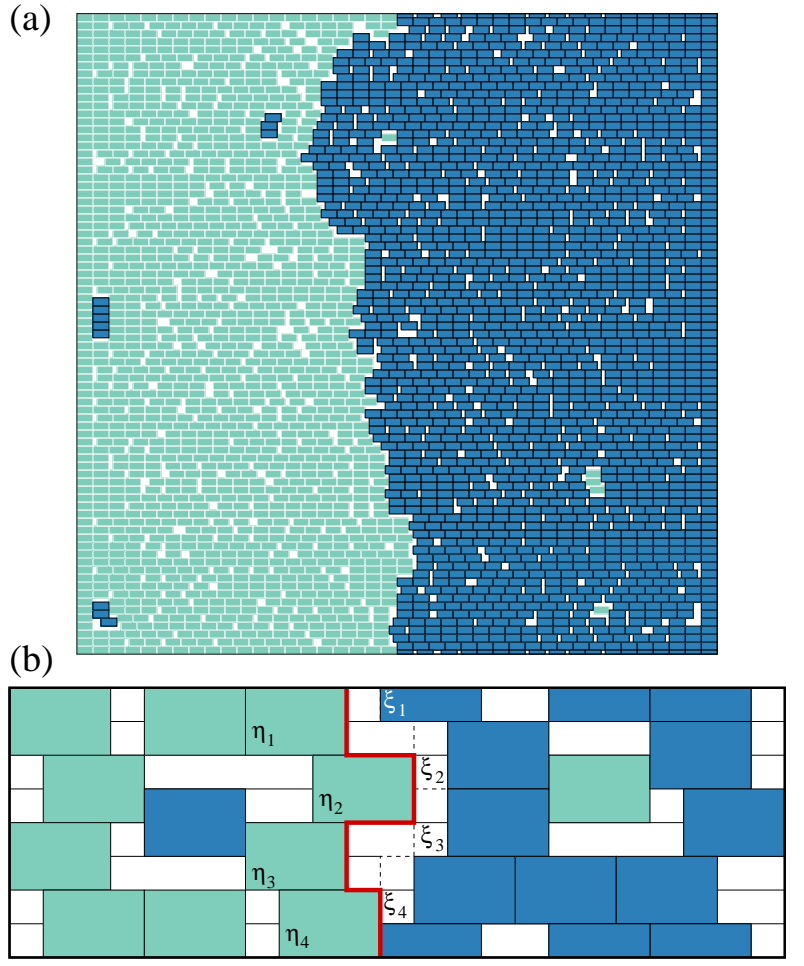

Fig. 1: (a) Snapshot of a typical configuration of $2 \times 4$ rectangles where the left (right) phase is constrained to be even (odd) by fixing the rectangles at the boundary. Even (odd) rectangles are shown in teal (blue). The system size is $160 \times 160$. (b) A schematic diagram of the interface (thick red line) between two phases having a defect each. $\eta_{i}$ is the x-coordinate of the head of the rightmost non-defect rectangle in row $i$, and $\xi_{i}=\max \left(\eta_{i}, \eta_{i-1}\right)+d$ is the minimum allowed $x$-coordinate for heads of odd rectangle on the $(2 i-1)$ th row.

interface $I$, where $z_{D}$ is the activity of a defect, and $e$ and $o$ refer to even and odd respectively. Then, $\Omega_{e o}\left(z, z_{D}\right)$, the partition function with an interface present, is the sum of $\Omega_{e o}\left(z, z_{D} \mid I\right)$ over different interfaces $I$ :

$$
\Omega_{e o}\left(z, z_{D}\right)=\sum_{I} \Omega_{e o}\left(z, z_{D} \mid I\right) .
$$

Let $\Omega_{e e}\left(z, z_{D}\right)$ be the partition function when all rectangles at the boundaries are fixed to be even (no interface). Then,

$$
\sigma(z)=\lim _{M \rightarrow \infty} \frac{-1}{M} \ln \left[\frac{\Omega_{e o}\left(z, z_{D}\right)}{\Omega_{e e}\left(z, z_{D}\right)}\right],
$$

and we define the effective free energy $H_{\text {eff }}(I)$ of any interface $I$ to be

$$
H_{\mathrm{eff}}(I)=-\ln \left[\frac{\Omega_{e o}\left(z, z_{D} \mid I\right)}{\Omega_{e e}\left(z, z_{D}\right)}\right] .
$$

In the simplest approximation, we ignore overhangs such that the interface is a path from top to bottom with no upward steps allowed. Then, the interface $I$ is uniquely defined by $\{\eta\}$, where $\eta_{i}$ is the head of the right most rectangle of the even phase [see Fig 1(b)]. Since the interactions are hard core, given an interface $I$, the left and right phases may be occupied with rectangles independent of each other, such that $\Omega_{e o}\left(z, z_{D} \mid I\right)=$ $\Omega_{L}\left(z, z_{D} \mid I\right) \Omega_{R}\left(z, z_{D} \mid I\right)$, where $L(R)$ refers to left (right) phase.

Each of the partition functions $\Omega_{e e}\left(z, z_{D}\right), \Omega_{L}\left(z, z_{D} \mid I\right)$ and $\Omega_{R}\left(z, z_{D} \mid I\right)$, as well as the effective free energy $H_{\text {eff }}(I)$ may be expanded as a perturbation series in $z_{D}$. For example, $\Omega_{e e}\left(z, z_{D}\right)=\Omega_{e e}^{(0)}(z)+z_{D} \Omega_{e e}^{(1)}(z)+O\left(z_{D}^{2}\right)$, where the superscript $(n)$ refers to the presence of $n$ defects. Expanding Eqs. (21) and (3) in powers of $z_{D}$, we obtain the zero defect contribution to $H_{\mathrm{eff}}(I)$ and $\sigma(z)$ to be

$$
\begin{aligned}
H_{\mathrm{eff}}^{(0)}(I) & =-\ln \frac{\Omega_{L}^{(0)}(z \mid I) \Omega_{R}^{(0)}(z \mid I)}{\Omega_{e e}^{(0)}(z)}, \\
\sigma^{(0)}(z) & =\lim _{M \rightarrow \infty} \frac{-1}{M} \ln \sum_{I} e^{-H_{\mathrm{eff}}^{(0)}(I)} .
\end{aligned}
$$

Details of this expansion may be found in Ref. [54].

Zero defect calculation. - The calculation of $\sigma^{(0)}(z)$ is quite straightforward. In the absence of defects, each phase consists of only rectangles of one kind, either even or odd. This implies that the configuration of rectangles in a row is independent of those in the other rows. Thus, the partition functions $\Omega_{e e}^{(0)}(z), \Omega_{L}^{(0)}(z \mid I)$ and $\Omega_{R}^{(0)}(z \mid I)$ become products of one-dimensional partition functions, and we obtain

$$
\begin{aligned}
\Omega_{e e}^{(0)}(z) & =\left[\omega_{0}(N)\right]^{M / 2}, \\
\Omega_{L}^{(0)}(z \mid I) & =\prod_{i=1}^{M / 2}\left[z \omega_{0}\left(\eta_{i}\right)\right], \\
\Omega_{R}^{(0)}(z \mid I) & =\prod_{i=1}^{M / 2} \omega_{0}\left(N-\xi_{i}\right),
\end{aligned}
$$

where the interface is specified by $\{\eta\}, \xi_{i}=$ $\max \left(\eta_{i}, \eta_{i-1}\right)+d$ [see Fig. 1(b)], and $\omega_{0}(N)$ is the partition function of a system of hard rods of length $d$ on a one dimensional open chain of length $N$. The factor $z$ in Eq. (7) accounts for the constraint that the right most site of each even row in the even phase should be occupied. It is easy to see that $\omega_{0}$ obeys the recursion relation

$$
\omega_{0}(\ell)=z \omega_{0}(\ell-d)+\omega_{0}(\ell-1),
$$

for $\ell=1,2, \ldots$ with $\omega_{0}(0)=1$, and $\omega_{0}(\ell)=0$ for $\ell<0$. For large $N, \omega_{0}(N)=a \lambda^{N}[1+O(\exp (-N))]$, where $\lambda$ is the largest root of the equation

$$
\lambda^{d}-\lambda^{d-1}=z .
$$

It is easily shown that (see Ref. [54])

$$
\begin{aligned}
a & =\frac{\lambda}{d(\lambda-1)+1}, \\
\sum_{j} \omega_{0}(j) x^{j} & =\frac{1}{1-x-z x^{d}} .
\end{aligned}
$$


The effective (free) energy of the interface $\{\eta\}$ is then [see Eq. (4)]

$$
H_{\mathrm{eff}}^{(0)}=\frac{-M}{2} \ln \left[a\left(1-\lambda^{-1}\right)\right]+\frac{1}{2} \ln \lambda \sum_{i}\left|\eta_{i+1}-\eta_{i}\right|,
$$

and we obtain the interfacial tension when there are no defects [see Eq. (5)] to be

$$
\sigma^{(0)}(z)=-\frac{1}{2} \ln \left[\frac{(\sqrt{\lambda}+1)^{2}}{d(\lambda-1)+1}\right]
$$

where $\lambda$ is related to $z$ by Eq. (10).

The interface $\{\eta\}$ is a PDSAW, a self avoiding walk in which steps in the upward direction are disallowed. From Eq. (13), we can identify the weights of steps in left, right and down directions to be $u=\lambda^{-1 / 2}, v=\lambda^{-1 / 2}$, and $w=a\left(1-\lambda^{-1}\right)$ respectively. The generating function of such a PDSAW is easily seen to be

$$
G=\left[1-w-\frac{w u}{1-u}-\frac{w v}{1-v}\right]^{-1}
$$

The phase transition occurs when the interfacial tension is zero or equivalently when the generating function $G$ in Eq. (15) diverges. Setting $\sigma^{(0)}(z)=0$, we obtain the estimates of the critical parameters $\lambda_{c}^{*}(d)$ and $\rho_{c}^{*}(d)$ to be

$$
\begin{aligned}
\sqrt{\lambda_{c}^{*}(d)} & =\frac{1+\sqrt{1-d+d^{2}}}{d-1} \\
\rho_{c}^{*}(d) & =\frac{d\left(1+d+2 \sqrt{1-d+d^{2}}\right)}{\left(d+\sqrt{1-d+d^{2}}\right)^{2}},
\end{aligned}
$$

and $z_{c}^{*}(d)=\lambda_{c}^{*}(d)^{d}-\lambda_{c}^{*}(d)^{d-1}$. For hard-squares $(d=$ $2), \lambda_{c}^{*}(2)=(\sqrt{3}+1)^{2}, z_{c}^{*}(2)=(4+2 \sqrt{3})(3+2 \sqrt{3}) \approx$ $48.25, \rho_{c}^{*}(2)=(6+4 \sqrt{3})(7-4 \sqrt{3}) \approx 0.928$. These should be compared with numerically obtained $z_{c}=97.5$ and $\rho_{c}=0.932$ 22, 44, 45. For large $d$ limit, the solution is $\lambda_{c}^{*}=1+3 d^{-1}+O\left(d^{-2}\right), z_{c}=60.26 d^{-1}+O\left(d^{-2}\right)$, and $\rho_{c}^{*}=0.75+0.375 d^{-1}+O\left(d^{-2}\right)$. These should be compared with the numerically obtained values $z_{c} \approx 71.0 / d$ and $\rho_{c} \approx$ $0.73+0.46 / d$ [31,48]. For intermediate values of $d$, the analytical expression is compared with the Monte Carlo results in Fig. 2. We note that the results of calculation with no defects are already significantly better than earlier estimates.

One defect calculation. - Consider now the corrections to the interfacial tension to the first order in $z_{D}$. For this, we need to compute $\Omega_{e e}^{(1)}, \Omega_{L}^{(1)}(z \mid I)$, and $\Omega_{R}^{(1)}(z \mid I)$, the partition functions when a single defect is present. There are configurations which may be considered as single odd defect in the even phase, or equally as a single even defect in the odd phase by repositioning the interface [see Fig. 3(a)]. To prevent double counting, we adopt the convention that a defect in the even phase should have at least two even rectangles to its right. Else, we redefine the interface locally.

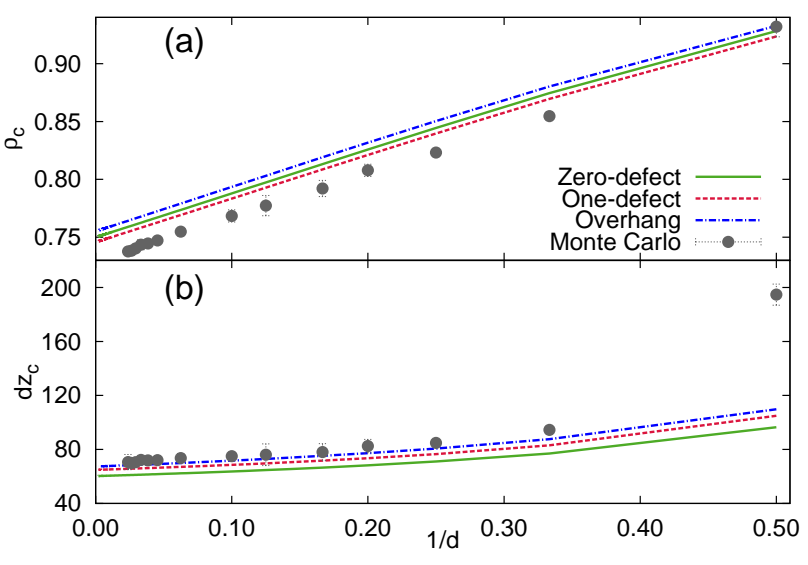

Fig. 2: The results obtained for (a) critical density $\rho_{c}$ and (b) critical activity $z_{c}$ for the disordered-columnar transition with zero defect (green line), one defect (red line) and overhangs (blue line) are compared with the results from Monte Carlo simulations (data points). The Monte Carlo data for $d>16$ is from Ref. [4], the data for $d \leq 16$ has been generated using the algorithm introduced in Refs. 27,55, and the data for $d=2$ is from Refs. 22, 44,45 .

A defect rectangle affects the occupation of utmost the two rows adjacent to it, by splitting the row(s) into two intervals that may be occupied independent of each other. However, unlike the zero defect case, the intervals may be small instead of being extensive in $N$, and therefore it is necessary to know $\omega_{0}(\ell)$ for all $\ell$ and not just for large $\ell$. The detailed calculation of the contribution from one defect may be found in Ref. 54. The one defect calculation reduces to finding the expectation values of two properties of the PDSAW: the mean step length and two-point correlations between adjacent steps.

By equating $\sigma(z)=\sigma^{(0)}(z)+z_{D} \sigma^{(1)}(z)$ to zero we obtain

$$
\begin{aligned}
& z_{c}^{*}(d)=65.23 d^{-1}+O\left(d^{-2}\right) \\
& \rho_{c}^{*}(d)=0.746+0.374 d^{-1}+O\left(d^{-2}\right) .
\end{aligned}
$$

For hard squares, we obtain $z_{c}^{*}(2)=52.49$ and $\rho_{c}^{*}(2)=$ 0.923 . These results and those for general $d$ are compared with the Monte Carlo results as well as the contribution from zero defects in Fig. 2. The one defect calculation improves the estimates for $z_{c}(d)$ and $\rho_{c}(d)$ for all $d>2$. For $d=2$, while $z_{c}(2)$ increases towards the Monte Carlo results, $\rho_{c}(2)$ decreases slightly away from the Monte Carlo results.

Calculation including overhangs. - Another improvement over the zero defect calculation would be to include in the sum over interfaces, interfaces with overhangs. In this calculation, we assume perfect columnar order in each of the phases to the left or right of interface. We consider all interfaces in which the overhangs have height at most 1 . These interfaces may be defined as 
(a)

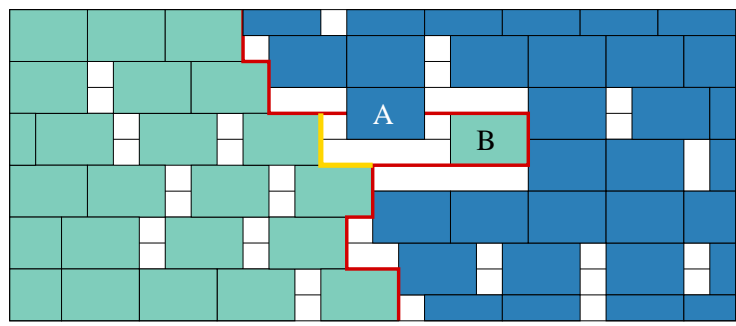

(b)

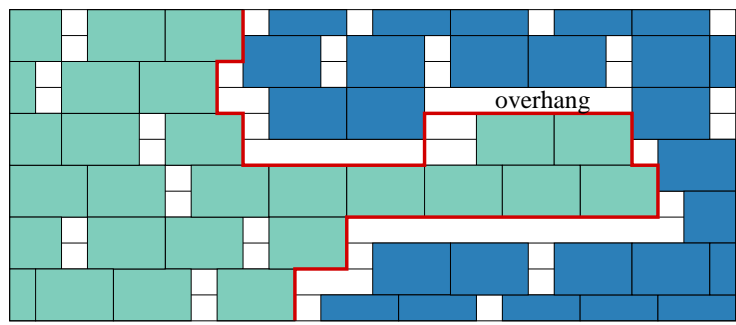

Fig. 3: (a) A configuration with a single defect where the interface may be drawn (red or yellow) so that the defect is an odd rectangle (A) in the even phase, or an even rectangle (B) in the odd phase. (b) A configuration in which the interface has one overhang.

self-avoiding walks such that if the walk once reaches the layer $y$ it never goes to the layer $y-4$ any time later, but may go back to $y-2$, a single vertical step of the walk being of length two lattice spacings. An interface with one overhang of height 1 is shown in Fig. 3(b).

If we denote the up, right, down, and left steps of the walk by letters $U, R, D$, and $L$ respectively, it is easily seen that an overhang configuration consists of sequence of steps of the type $R^{a} U R^{b} D$, where $a, b>d$. Define

$$
\begin{aligned}
W_{R} & =\sum_{a, b \geq d}^{\infty} R^{a} U R^{b} D, \\
W_{R}^{*} & =W_{R}+W_{R} W_{R}+W_{R} W_{R} W_{R}+\ldots,
\end{aligned}
$$

and a similar definition for sum over overhang configurations to the left $W_{L}^{*}$. $W_{R}^{*}$ and $W_{L}^{*}$ generate overhangs of height 1.

It is straight forward to write down the sum over configurations of rectangles corresponding to an interface with such overhangs as a product of one-dimensional partition functions $\omega_{0}$. Note that in the definition of $W_{R}$, there is a sum over lengths of steps $a$ and $b$. These come with a weight $\lambda^{-3(a+b) / 2}$. This implies that the corresponding weight is expressed in terms of the function $\sum_{i} \omega_{0}(i) \lambda^{-3 i / 2}$, which is a simple rational function of $\lambda^{-3 / 2}$ and $z$ [see Eq. (12)], and this simplifies the expressions of weights of $W_{R}$ and $W_{R}^{*}$ enormously. Details of the calculation are given in Ref. [54. Equating the surface tension to zero, we obtain the estimate

$$
\begin{aligned}
& z_{c}^{* *}(d)=67.78 d^{-1}+O\left(d^{-2}\right), \\
& \rho_{c}^{* *}(d)=0.755+0.382 d^{-1}+O\left(d^{-2}\right),
\end{aligned}
$$

and $z_{c}^{* *}(2)=54.87$ and $\rho_{c}^{* *}(2)=0.9326$. These are an improvement over the estimates of $z_{c}(d)$ from the one defect calculations [see Fig. 2 for comparison]. However, the calculation of overhangs does not affect the bulk quantities, and therefore the estimates for critical density $\rho_{c}(d)$ increase. While the estimates for $\rho_{c}(d)$ matches with the Monte Carlo result for $d=2$, it increases away from the Monte Carlo results for $d>2$.

Discussion. - In this paper, we determined the critical activity and critical density of transition between the nematic and columnar phases in the problem of $2 \times d$ oriented rectangles on a square lattice approximately, by estimating the interfacial tension between two differently ordered columnar phases. This was done in two ways: first in terms of a perturbation series in number of defects, and second by including a subset of overhangs in the interface. Keeping only the first order terms in the expansion in the first case and overhangs of height 1 in the second case, we obtain estimates that are in fair agreement with Monte Carlo results and an improvement over earlier estimates. For example, when $d=2$, Monte Carlo estimates are $z_{c}(2) \approx 97.5$, and $\rho_{c}(2) \approx 0.932$, while we obtain $z_{c}(2)=52.49$ and 54.87 , and $\rho_{c}(2)=0.923$ and 0.932. These should be compared with estimates from high density expansion $\left(z_{c}(2)=14.86\right)$ [16, 18, a lattice version of density functional theory $\left(z_{c}(2)=11.09\right)$ [4/,56 an approximate counting method using transfer matrices $\left(z_{c}(2)=11.09\right)$ [57, a Flory type mean-field theory (with similar prediction) [58, and cluster variational method $\left(z_{c}(2)=17.22\right)[16]$. A calculation of interfacial tension in the next nearest neighbour Ising antiferromagnet, gives a better estimate $z_{c}(2)=135.9$ [46], still off by about $40 \%$. For the critical density, the high density expansion gives $\rho_{c}(2)=0.75$, cluster variation method gives $\rho_{c}(2)=0.80$, and the transfer matrix calculations $\rho_{c}(2)=0.76$. Likewise, we obtain significantly better estimates for the critical parameters for the hard rectangle gas also. These may be systematically improved by including more defects or overhangs. Also, the calculations may be extended to a system of rectangles of same width and different length:1. Similar results may be derived for mixtures of squares and dimers [38.

The interfacial tension was calculated for a system with only horizontal rectangles. However, it is straightforward to show that for large $d$, adding vertical rectangles introduces only exponentially small (in $d$ ) corrections to the critical density. For $d=2$, this approximation is irrelevant. However, for small $d>2$, the effects are not negligible [28, 32].

On general grounds, the nematic-columnar transition is expected to be in the Ising universality class [28, 32]. In our calculation, the interfacial tension varies as $\left|z-z_{c}\right|$ near the transition point, consistent with the behavior in 
the Ising universality class 59 .

$$
* * *
$$

The simulations were done on the supercomputer Annapurna at the Institute of Mathematical Sciences. DD was supported in part by the Department of Science and Technology (India) under the grant DST-SR/S2/JCB$24 / 2005$.

\section{REFERENCES}

[1] Pusey P. N. and van Megan W., Nature, 320 (1986) 340.

[2] Alder B. J. and Wainwright T. E., J. Chem. Phys., 27 (1957) 1208

[3] Hoover W. G. and Ree F. H., J. Chem. Phys., 49 (1968) 3609

[4] De Gennes P. and Prost J., The physics of liquid crystals (Oxford University Press) 1995.

[5] Onsager L., Ann. N.Y. Acad. Sci., 51 (1949) 627.

[6] Bolnuis P. and Frenkel D., J. Chem. Phys., 106 (1997) 666.

[7] Flory P. J., Proc. R. Soc., 234 (1956) 73.

[8] Zwanzig R., J. Chem. Phys., 39 (1963) 1714.

[9] Taylor D. E., Williams E. D., Park R. L., Bartelt N. C. and Einstein T. L., Phys. Rev. B, 32 (1985) 4653.

[10] Kasteleyn P. W., Physica, 27 (1961) 1209.

[11] Heilmann O. J. and Lieb E., Commun. Math. Phys., 25 (1972) 190.

[12] Dhar D. and Chandra S., Phys. Rev. Lett., 100 (2008) 120602.

[13] Ghosh A., Dhar D. and Jacobsen J. L., Phys. Rev. E, 75 (2007) 011115.

[14] Baxter R. J., J. Phys. A, 13 (1980) L61.

[15] Verberkmoes A. and Nienhuis B., Phys. Rev. Lett., 83 (1999) 3986

[16] Bellemans A. and Nigam R. K., J. Chem. Phys., 46 (1967) 2922.

[17] Pearce P. A. and Seaton K. A., J. Stat. Phys., 53 (1988) 1061.

[18] Ramola K. and Dhar D., Phys. Rev. E, 86 (2012) 031135.

[19] Wojciechowski K. and Frenkel D., Comput. Meth. Sci. Technol., 10 (2004) 235.

[20] Zhao K., Bruinsma R. and Mason T. G., Proc. Natl. Acad. Sci. U.S.A., 108 (2011) 2684.

[21] Avendaño C. and Escobedo F. A., Soft Matter, 8 (2012) 4675 .

[22] Fernandes H. C. M., Arenzon J. J. and Levin Y., J. Chem. Phys., 126 (2007) 114508.

[23] Nath T. and Rajesh R., Phys. Rev. E, 90 (2014) 012120.

[24] Barnes B. C., Siderius D. W. and Gelb L. D., Langmuir, 25 (2009) 6702.

[25] Ghosh A. and Dhar D., Europhys. Lett., 78 (2007) 20003.

[26] Dhar D., Rajesh R. and Stilck J. F., Phys. Rev. E, 84 (2011) 011140

[27] Kundu J., Rajesh R., Dhar D. and Stilck J. F., Phys. Rev. E, 87 (2013) 032103.
[28] Kundu J. and Rajesh R., Phys. Rev. E, 89 (2014) 052124.

[29] Zhao K., Harrison C., Huse D., Russel W. B. and Chaikin P. M., Phys. Rev. E, 76 (2007) 040401.

[30] Donev A., Burton J., Stillinger F. H. and Torquato S., Phys. Rev. B, 73 (2006) 054109.

[31] Kundu J. and Rajesh R., Phys. Rev. E, 91 (2015) 012105.

[32] Kundu J. and Rajesh R., Euro. Phys. J. B, 88 (2015) 133.

[33] Edlund E. and Jасовi M. N., Phys. Rev. Lett., 105 (2010) 137203.

[34] Alet F., Ikhlef Y., Jacobsen J. L., Misguich G. and Pasquier V., Phys. Rev. E, 74 (2006) 041124.

[35] Jin S., Sen A. and Sandvik A. W., Phys. Rev. Lett., 108 (2012) 045702.

[36] Papanikolaou S., Luijten E. and Fradkin E., Phys. Rev. B, 76 (2007) 134514

[37] Wenzel S., Coletta T., Korshunov S. E. and Mila F., Phys. Rev. Lett., 109 (2012) 187202.

[38] Ramola K., Damle K. and Dhar D., Phys. Rev. Lett., 114 (2015) 190601

[39] Peierls R., Proc. Cam. Phil. Soc., 32 (1936) 477.

[40] Dobrushin R., Funct. Anal. Appl., 2 (1968) 302.

[41] Heilmann O. J. and Praestgaard E., J. Phys. A, 7 (1974) 1913.

[42] Disertori M. and Giuliani A., Commun. Math. Phys., 323 (2013) 143.

[43] Bellemans A. and Nigam R. K., Phys. Rev. Lett., 16 (1966) 1038

[44] Feng X., Blöte H. W. J. and Nienhuis B., Phys. Rev. E, 83 (2011) 061153.

[45] Zhitomirsky M. E. and Tsunetsugu H., Phys. Rev. B, 75 (2007) 224416

[46] Slotte P. A., J. Phys. C, 16 (1983) 2935.

[47] Lafuente L. and Cuesta J. A., J. Chem. Phys., 119 (2003) 10832.

[48] Nath T., Kundu J. and Rajesh R., J. Stat. Phys., 160 (2015) 1173

[49] Tonks L., Phys. Rev., 50 (1936) 955.

[50] Takahashi H., Proc. Phys.-Math. Soc. Japan, 24 (1942) 60.

[51] Müller-Hartmann E. and Zittartz J., Z. Phys. B Condens Matter, 27 (1977) 261.

[52] Abraham D. B. and Reed P., Phys. Rev. Lett., 33 (1974) 377.

[53] Selke W. and Pesch W., Z. Phys. B Condens Matter, 47 (1982) 335

[54] NATh T., DhaR D. and RAJEsh R., arXiv preprint arXiv:1601.02198, (2016) .

[55] Kundu J., Rajesh R., Dhar D. and Stilck J. F., AIP Conf. Proc., 1447 (2012) 113.

[56] Lafuente L. and Cuesta J. A., J. Phys.: Condens. Matter, 14 (2002) 12079.

[57] Temperley H., Proc. Phys. Soc., 77 (1961) 630.

[58] Marques Fernandes H. C., Levin Y. and Arenzon J. J., Phys. Rev. E, 75 (2007) 052101.

[59] McCoy B. M. and Wu T. T., The two-dimensional Ising model (Courier Corporation) 2014. 
Supplemental Information for Stability of columnar order in assemblies of hard rectangles or squares

Trisha Nath, Deepak Dhar, and R. Rajesh 


\section{EXPRESSIONS FOR ONE-DEFECT CONTRIBUTION TO $H_{\mathrm{eff}}(I)$ AND $\sigma(z)$}

We Taylor expand $\Omega_{e e}\left(z, z_{d}\right)$ in powers of $z_{d}$, and write

$$
\Omega_{e e}\left(z, z_{d}\right)=\Omega_{e e}^{(0)}(z)+z_{d} \Omega_{e e}^{(1)}(z)+z_{D}^{2} \Omega_{e e}^{(2)}(z)+\ldots,
$$

and similar expressions for $\Omega_{L}(z \mid I), \Omega_{R}(z \mid I)$ etc. Using the definition of $H_{\text {eff }}(I)$ from Eq. (3) of main text, and expanding each partition functions in powers of $z_{d}$, we obtain

$$
\begin{aligned}
H_{\mathrm{eff}}^{(1)}(I) & =\frac{\Omega_{e e}^{(1)}(z)}{\Omega_{e e}^{(0)}(z)}-\frac{\Omega_{L}^{(1)}(z \mid I)}{\Omega_{L}^{(0)}(z \mid I)}-\frac{\Omega_{R}^{(1)}(z \mid I)}{\Omega_{R}^{(0)}(z \mid I)}, \\
\sigma^{(1)}(z) & =\lim _{M \rightarrow \infty} \frac{1}{M} \frac{\sum_{I} H_{\mathrm{eff}}^{(1)}(I) e^{-H_{\mathrm{eff}}^{(0)}(I)}}{\sum_{I} e^{-H_{\mathrm{eff}}^{(0)}}(I)}
\end{aligned}
$$

\section{DERIVATION OF EQ. (11)}

We start with Eq. (10) of main text. This equation has $d$ roots. Let them be denoted by $\lambda_{i}, i=1,2, \ldots, d$ where $\lambda_{1}$, the largest root, will be denoted by $\lambda$. Then for any $\ell$, the one-dimensional partition function can thus be written as

$$
\omega_{0}(\ell)=\sum_{i=1}^{d} a_{i} \lambda_{i}^{\ell} .
$$

For large $\ell$, we can approximate $\omega_{0}(\ell) \approx a \lambda^{\ell}$, where $a$ is $a_{1}$ in Eq. (44).

We calculate $a$ from the density of occupied sites. Suppose $\rho(d)$ is the density of the rectangles on the onedimensional chain of length $\ell$. Then $\rho(d)$ is given by

$$
\rho(d)=\frac{d}{\ell} z \frac{d}{d z} \ln \omega_{o}(\ell) .
$$

On substitution of $\omega_{0}(\ell) \approx a \lambda^{\ell}$ we obtain

$$
\rho(d)=\frac{d(\lambda-1)}{d(\lambda-1)+1} .
$$

Consider a site $m$ in the bulk of the chain. The probability that it is empty is $\left[\omega_{0}(m-1) \omega_{0}(1) \omega_{0}(\ell-m)\right] / \omega_{0}(\ell)$. Clearly this is equal to $1-\rho(d)$. Substituting $\omega_{0}(\ell) \approx a \lambda^{\ell}$ we obtain,

$$
1-\rho(d)=\frac{a}{\lambda}
$$

Substituting $\rho(d)$ from Eq. (6), we obtain $a$ as

$$
a=\frac{\lambda}{d(\lambda-1)+1} .
$$

\section{CALCULATION OF $\sigma^{(1)}(z)$}

Consider the contribution to the partition function when a single defect is present. Let the defect rectangle be placed such that it affects the occupation of rows $i$ and $i+1$, where $i=1, \ldots, M / 2$, with the $x$-coordinate of the head being denoted by $x$. Examples of defects for different boundary conditions are shown in Fig. 1(a)-(e). To avoid over-counting, we impose the constraints that a defect in the left phase should at least have two even rectangles to its right [see Fig. 1 (b)-(c)] and a defect in the right phase should have at least one odd rectangle to its left [see Fig. 1(d)-(e)]. In each of the cases shown in Fig. 1(a), (b), and (d), the defect splits the rows $i$ and $i+1$ into two 

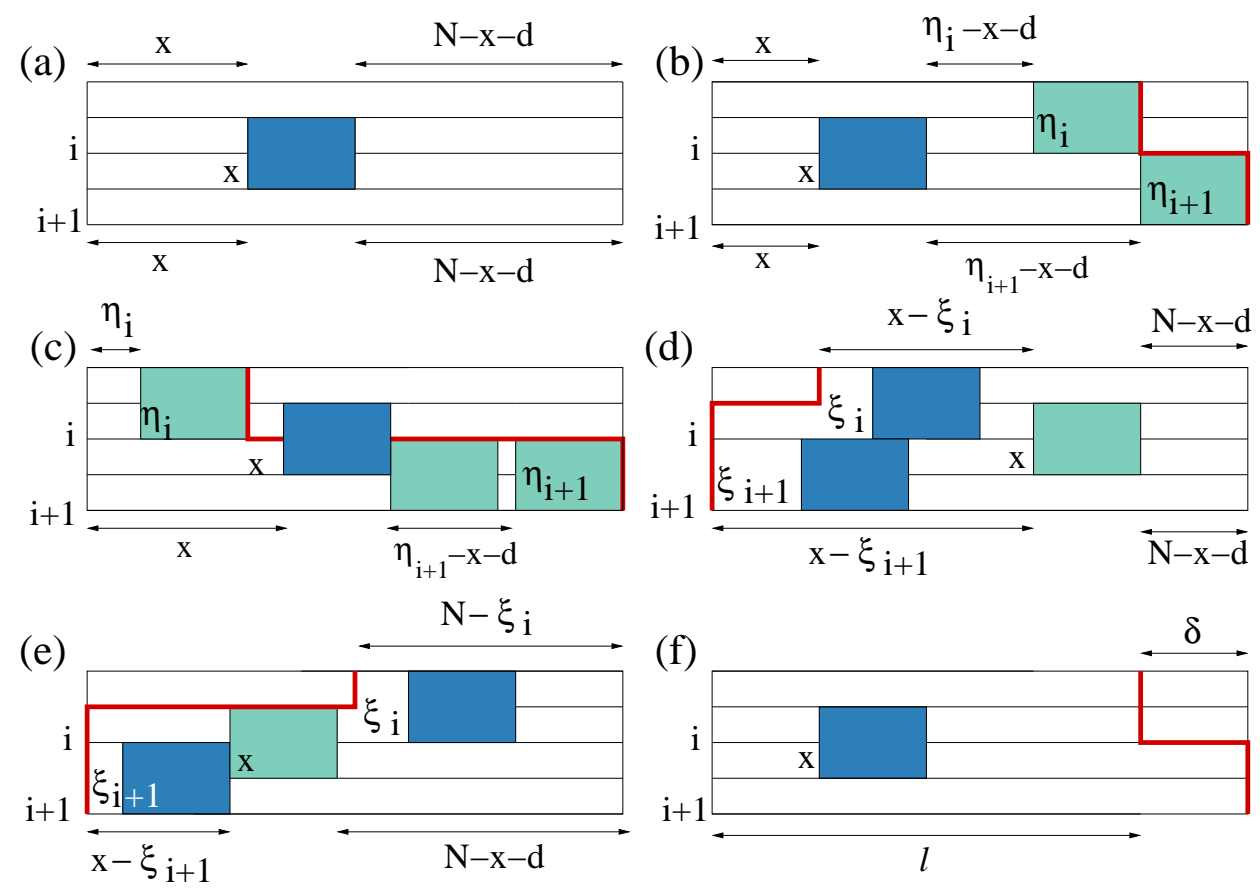

FIG. 1. Examples of single defects for (a) $\Omega_{e e}^{(1)}$, (b)-(c) $\Omega_{e o, L}^{(1)}$ and (d)-(e) $\Omega_{e o, R}^{(1)}$. (f) A generalized two-row system with a single defect in the bulk. The red lines denote the position of the interface.

intervals, the occupation of each interval being independent of the other. However in configurations of type shown in Fig. 1(c), and (e), the defect affects only one of the rows, splitting it into two open intervals. Thus, we obtain

$$
\begin{aligned}
& \frac{\Omega_{e e}^{(1)}}{\Omega_{e e}^{(0)}}=\frac{M}{2} \sum_{x=0}^{N-d}\left[\frac{\omega_{0}(x) \omega_{0}(N-x-d)}{\omega_{0}(N)}\right]^{2} \\
& \frac{\Omega_{e o, L}^{(1)}}{\Omega_{e o, L}^{(0)}}=\sum_{i=1}^{M / 2}\left[\sum_{x=0}^{x_{L}} \frac{\omega_{0}^{2}(x) \omega_{0}\left(\eta_{i}-x-d\right) \omega_{0}\left(\eta_{i+1}-x-d\right)}{\omega_{0}\left(\eta_{i}\right) \omega_{0}\left(\eta_{i+1}\right)}+\sum_{x=x_{L}+2 d}^{x_{L}^{\prime}-2 d} \frac{\omega_{0}(x)\left[\omega_{0}\left(x_{L}^{\prime}-x-d\right)-1\right]}{\omega_{0}\left(x_{L}^{\prime}\right)} \Theta\left(\left|\Delta \eta_{i}\right|-3 d\right)\right] \\
& \frac{\Omega_{e o, R}^{(1)}}{\Omega_{e o, R}^{(0)}}=\sum_{i=1}^{M / 2}\left[\sum_{x=x_{R}-d}^{N-d} \frac{\omega_{0}^{2}(N-x-d)\left[\omega_{0}\left(x-\xi_{i}\right) \omega_{0}\left(x-\xi_{i+1}\right)-1\right]}{\omega_{0}\left(N-\xi_{i}\right) \omega_{0}\left(N-\xi_{i+1}\right)}\right. \\
& \left.+\sum_{x=x_{R}^{\prime \prime}}^{x_{R}-d-1} \frac{\omega_{0}^{2}(N-x-d)\left[\omega_{0}\left(x-x_{R}^{\prime}\right)-1\right] \Theta\left(\left|\Delta \xi_{i}\right|-d-1\right)}{\omega_{0}\left(N-\xi_{i}\right) \omega_{0}\left(N-\xi_{i+1}\right)}+\sum_{x=x_{R}^{\prime}+d}^{x_{R}-2 d} \frac{\omega_{0}(N-x-d)\left[\omega_{0}\left(x-x_{R}^{\prime}\right)-1\right] \Theta\left(\left|\Delta \xi_{i}\right|-2 d\right)}{\omega_{0}\left(N-x_{R}^{\prime}\right)}\right]
\end{aligned}
$$

where $x_{L}=\min \left(\eta_{i}, \eta_{i+1}\right)-d, x_{L}^{\prime}=\max \left(\eta_{i}, \eta_{i+1}\right), x_{R}=\max \left(\xi_{i}, \xi_{i+1}\right)+d, x_{R}^{\prime}=\min \left(\xi_{i}, \xi_{i+1}\right), x_{R}^{\prime \prime}=\max \left(x_{R}-2 d+\right.$ $\left.1, x_{R}^{\prime}+d\right)$, and $\Theta(x)$ is a step function

$$
\Theta(x)= \begin{cases}1, & \text { if } x \geq 0 \\ 0, & \text { otherwise }\end{cases}
$$

and we have dropped the dependence on $z, I$ for notational simplicity. The first and second terms of Eq. (10) are contributions from Fig. 1(b) and (c) respectively. Similarly, the first term of Eq. (11) is contribution from Fig. 1(d), whereas the rest are contributions from configurations of the type Fig. प(e).

In the sums, when $x$ is not close to the lower or upper limits, we may substitute $\omega_{0}(\ell) \approx a \lambda^{\ell}$, else there are corrections due to the boundaries. To characterize these edge effects, we consider the partition function of a two-row system with a single defect and boundary as shown in Fig. [(f). The partition function of this shape for large $\ell$ is

$$
\sum_{x=0}^{\ell-d} \frac{\omega_{0}^{2}(x) \omega_{0}(\ell-x-d) \omega_{0}(\ell+\delta-x-d)}{\omega_{0}(\ell) \omega_{0}(\ell+\delta)}=\frac{a^{2}}{\lambda^{2 d}}[(\ell-d+1)+f(0)+f(\delta)],
$$


where $f(\delta)$ encodes the edge-effects,

$$
f(\delta)=\left[2 \sum_{p=2}^{d} \frac{a_{p}}{a} \frac{1}{1-\frac{\lambda_{p}}{\lambda}}\left(1+\left(\frac{\lambda_{p}}{\lambda}\right)^{|\delta|}\right)+\sum_{p, q=2}^{d} \frac{a_{p} a_{q}}{a^{2}} \frac{1}{1-\frac{\lambda_{p} \lambda_{q}}{\lambda^{2}}}\left(\frac{\lambda_{p}}{\lambda}\right)^{|\delta|}\right]
$$

Equations. (9), (10) and (11) may thus be simplified to

$$
\begin{aligned}
& \frac{\Omega_{e e}^{(1)}}{\Omega_{e e}^{(0)}}=\frac{M}{2} \frac{a^{2}}{\lambda^{2 d}}[(N-d+1)+2 f(0)], \\
& \frac{\Omega_{e o, L}^{(1)}}{\Omega_{e o, L}^{(0)}}=\frac{a^{2}}{\lambda^{2 d}} \sum_{i=1}^{M / 2}\left[x_{L}+1+f(0)+f\left(\Delta \eta_{i}\right)+p\left(\left|\Delta \eta_{i}\right|-3 d\right)\right], \\
& \frac{\Omega_{e o, R}^{(1)}}{\Omega_{e o, R}^{(0)}}=\frac{a^{2}}{\lambda^{2 d}} \sum_{i=1}^{M / 2}\left[N-x_{R}+1+f(0)+f\left(\Delta \xi_{i}\right)-\frac{a^{-2} \lambda^{-\left|\Delta \xi_{i}\right|}}{1-\lambda^{-2}}+p\left(\left|\Delta \xi_{i}\right|-2 d\right)+q\left(\left|\Delta \xi_{i}\right|\right)\right],
\end{aligned}
$$

where $\Delta \xi_{i}=\xi_{i+1}-\xi_{i}$, and $p(x)$ and $q(x)$ are defined as

$$
\begin{aligned}
p(x) & =\left[\frac{\lambda^{d}}{a}(x+1)-\frac{1-\lambda^{-x-1}}{a^{2}\left(1-\lambda^{-1}\right)}+\sum_{p=2}^{d} \frac{a_{p} \lambda_{p}^{d}}{a^{2}\left(1-\frac{\lambda_{p}}{\lambda}\right)}\left(1-\left(\frac{\lambda_{p}}{\lambda}\right)^{x+1}\right)\right] \Theta(x), \\
q(x) & =\frac{\Theta(x-d-1)}{a\left(1-\lambda^{-1}\right)}\left[-1+\frac{\lambda^{-x}}{a\left(1+\lambda^{-1}\right)}+\sqrt{\lambda}^{x-|x-2 d+1|-1}-\frac{\lambda^{-|x-2 d+1|-1}}{a\left(1+\lambda^{-1}\right)}\right. \\
& \left.+\sum_{p=2}^{d} \frac{a_{p}}{a} \frac{1-\lambda^{-1}}{1-\frac{\lambda_{p}}{\lambda^{2}}}\left(\frac{\lambda_{p}}{\lambda}\right)^{x}\left(\left(\frac{\lambda}{\lambda_{p}}\right)^{x-|x-2 d+1|-1}-1\right)\right] .
\end{aligned}
$$

The contribution from a single defect to the free energy of the interface, $H_{\mathrm{eff}}^{(1)}(I)$ [see Eq. (2)] may now be evaluated from Eqs. (15), (16) and (17):

$$
\begin{aligned}
H_{\mathrm{eff}}^{(1)}(I) & =\frac{a^{2}}{\lambda^{2 d}} \sum_{i=1}^{M / 2}\left[2 d-1+\frac{2\left|\Delta \eta_{i}\right|+\left|\Delta \xi_{i}\right|}{2}+\frac{a^{-2} \lambda^{-\left|\Delta \xi_{i}\right|}}{1-\lambda^{-2}}-f\left(\Delta \eta_{i}\right)-p\left(\left|\Delta \eta_{i}\right|-3 d\right)-f\left(\Delta \xi_{i}\right)\right. \\
& \left.-p\left(\left|\Delta \xi_{i}\right|-2 d\right)-q\left(\left|\Delta \xi_{i}\right|\right)\right] .
\end{aligned}
$$

Substituting Eq. (20) in Eq. (3) we obtain interfacial tension with one defect as

$$
\begin{aligned}
\sigma^{(1)}(z) & =\frac{a^{2}}{M \lambda^{2 d}} \sum_{i=1}^{M / 2}\left[2 d-1+\frac{\sqrt{\lambda}-1}{\sqrt{\lambda}+1} \sum_{\Delta \eta_{i}}\left(\left|\Delta \eta_{i}\right|-f\left(\Delta \eta_{i}\right)-p\left(\left|\Delta \eta_{i}\right|-3 d\right)\right) \sqrt{\lambda}^{-\left|\Delta \eta_{i}\right|}\right. \\
& \left.+\left(\frac{\sqrt{\lambda}-1}{\sqrt{\lambda}+1}\right)^{2} \sum_{\Delta \eta_{i}, \Delta \eta_{i+1}}\left(\frac{\left|\Delta \xi_{i}\right|}{2}-f\left(\Delta \xi_{i}\right)+\frac{a^{-2} \lambda^{-\left|\Delta \xi_{i}\right|}}{1-\lambda^{-2}}-p\left(\left|\Delta \xi_{i}\right|-2 d\right)-q\left(\left|\Delta \xi_{i}\right|\right)\right) \sqrt{\lambda}^{-\left|\Delta \eta_{i}\right|-\left|\Delta \eta_{i+1}\right|}\right] .
\end{aligned}
$$


Summing over $\Delta \eta$ and $\Delta \xi \mathrm{s}$, each of the terms in Eq. (21) are evaluated to be

$$
\begin{aligned}
& \frac{\sqrt{\lambda}-1}{\sqrt{\lambda}+1} \sum_{\Delta \eta_{i}}\left|\Delta \eta_{i}\right| \sqrt{\lambda}^{-\left|\Delta \eta_{i}\right|}=\frac{2 \sqrt{\lambda}}{\lambda-1} \\
& \left(\frac{\sqrt{\lambda}-1}{\sqrt{\lambda}+1}\right)^{2} \sum_{\Delta \eta_{i}, \Delta \eta_{i+1}} \frac{\left|\tilde{\xi}_{i}\right|}{2} \sqrt{\lambda}^{-\left|\Delta \eta_{i}\right|-\left|\Delta \eta_{i+1}\right|}=\frac{\sqrt{\lambda}+\lambda+\lambda^{3 / 2}}{(\sqrt{\lambda}-1)(\sqrt{\lambda}+1)^{3}}, \\
& \frac{a^{-2}}{1-\lambda^{-2}}\left(\frac{\sqrt{\lambda}-1}{\sqrt{\lambda}+1}\right)^{2} \sum_{\Delta \eta_{i}, \Delta \eta_{i+1}} \sqrt{\lambda}^{-2\left|\Delta \xi_{i}\right|-\left|\Delta \eta_{i}\right|-\left|\Delta \eta_{i+1}\right|}=\frac{\lambda^{2}}{a^{2}(\sqrt{\lambda}-1)(\sqrt{\lambda}+1)^{3}} \\
& \frac{\sqrt{\lambda}-1}{\sqrt{\lambda}+1} \sum_{\Delta \eta_{i}} f\left(\Delta \eta_{i}\right) \sqrt{\lambda}^{-\left|\Delta \eta_{i}\right|}=2 \sum_{p=2}^{d} \frac{a_{p}\left(\lambda^{2}-\lambda_{p}\right)}{a\left(1-\frac{\lambda_{p}}{\lambda}\right)(\sqrt{\lambda}+1)\left(\lambda^{3 / 2}-\lambda_{p}\right)} \\
& +\sum_{p, q=2}^{d} \frac{a_{p} a_{q}(\sqrt{\lambda}-1)\left(\lambda^{3}-\lambda_{p} \lambda_{q}\right)}{a^{2}\left(1-\frac{\lambda_{p} \lambda_{q}}{\lambda^{2}}\right)(\sqrt{\lambda}+1)\left(\lambda^{3 / 2}-\lambda_{p}\right)\left(\lambda^{3 / 2}-\lambda_{q}\right)}, \\
& \left(\frac{\sqrt{\lambda}-1}{\sqrt{\lambda}+1}\right)^{2} \sum_{\Delta \eta_{i}, \Delta \eta_{i+1}} f\left(\Delta \xi_{i}\right) \sqrt{\lambda}^{-\left|\Delta \eta_{i}\right|-\left|\Delta \eta_{i+1}\right|}=2 \sum_{p=2}^{d} \frac{a_{p}\left(2 \lambda^{2}+2 \lambda^{5 / 2}+\lambda^{3}-\lambda_{p}(1+2 \sqrt{\lambda}+2 \lambda)\right)}{a\left(1-\frac{\lambda_{p}}{\lambda}\right)(\sqrt{\lambda}+1)^{3}\left(\lambda^{3 / 2}-\lambda_{p}\right)} \\
& +\sum_{p, q=2}^{d} \frac{a_{p} a_{q}\left(\lambda^{3}\left(\lambda^{3 / 2}+\lambda+\sqrt{\lambda}-1\right)-\lambda^{2}\left(\lambda_{p}+\lambda_{q}\right)(1+\sqrt{\lambda})+\lambda_{p} \lambda_{q}\left(1+\sqrt{\lambda}+\lambda-\lambda^{3 / 2}\right)\right)}{a^{2}\left(1-\frac{\lambda_{p} \lambda_{q}}{\lambda^{2}}\right)(\sqrt{\lambda}+1)^{3}\left(\lambda^{3 / 2}-\lambda_{p}\right)\left(\lambda^{3 / 2}-\lambda_{q}\right)}, \\
& \left.\frac{\sqrt{\lambda}-1}{\sqrt{\lambda}+1} \sum_{\Delta \eta_{i}} p\left(\left|\Delta \eta_{i}\right|-3 d\right)\right) \sqrt{\lambda}^{-\left|\Delta \eta_{i}\right|}=\frac{2 \lambda^{(1-3 d) / 2}\left(a(1-3 d) \lambda^{d}-\frac{\lambda}{\lambda-1}\right)}{a^{2}(\sqrt{\lambda}+1)}+\frac{2 \lambda^{(1-d) / 2}(1+3 d(\sqrt{\lambda}-1))}{a(\lambda-1)} \\
& +\frac{2 \lambda^{-3(d-1) / 2}}{a^{2}(\sqrt{\lambda}+1)^{2}\left(\lambda^{3 / 2}-1\right)}+2 \sum_{p=2}^{d} \frac{a_{p} \lambda^{-3(d-1) / 2} \lambda_{p}^{d}}{a^{2}\left(\lambda-\lambda_{p}\right)(\sqrt{\lambda}+1)}\left(1-\frac{(\sqrt{\lambda}-1) \lambda_{p}}{\lambda^{3 / 2}-\lambda_{p}}\right)
\end{aligned}
$$

$$
\begin{aligned}
\left(\frac{\sqrt{\lambda}-1}{\sqrt{\lambda}+1}\right)^{2} \sum_{\Delta \eta_{i}, \Delta \eta_{i+1}} p\left(\left|\Delta \xi_{i}\right|-2 d\right) & \sqrt{\lambda}^{-\left|\Delta \eta_{i}\right|-\left|\Delta \eta_{i+1}\right|}=\frac{2 \lambda^{(1-2 d) / 2}(1+\sqrt{\lambda}+\lambda)\left(a(1-2 d) \lambda^{d}-\frac{\lambda}{\lambda-1}\right)}{a^{2}(\sqrt{\lambda}+1)^{3}} \\
& +\frac{2 \lambda^{(3-2 d) / 2}}{a^{2}(\sqrt{\lambda}-1)(\sqrt{\lambda}+1)^{4}}+\frac{2 \sqrt{\lambda}(1+\sqrt{\lambda}+\lambda)(1+2 d(\sqrt{\lambda}-1))}{a(\sqrt{\lambda}+1)^{3}(\sqrt{\lambda}-1)} \\
& +2 \sum_{p=2}^{d} \frac{a_{p} \lambda^{(3-2 d) / 2} \lambda_{p}^{d}(1+\sqrt{\lambda}+\lambda)}{a^{2}\left(\lambda-\lambda_{p}\right)(\sqrt{\lambda}+1)^{3}}\left(1-\frac{(\sqrt{\lambda}-1) \lambda_{p}}{\lambda^{3 / 2}-\lambda_{p}}\right)
\end{aligned}
$$




$$
\begin{aligned}
& \left(\frac{\sqrt{\lambda}-1}{\sqrt{\lambda}+1}\right)^{2} \sum_{\Delta \eta_{i}, \Delta \eta_{i+1}} q\left(\left|\Delta \xi_{i}\right|\right) \sqrt{\lambda}^{-\left|\Delta \eta_{i}\right|-\left|\Delta \eta_{i+1}\right|}=\frac{2 \lambda^{2-\frac{3 d}{2}}}{a^{2}(\sqrt{\lambda}+1)^{3}\left(\lambda^{2}-1\right)}-\frac{2(\lambda+\sqrt{\lambda}+1) \lambda^{1-\frac{d}{2}}}{a(\sqrt{\lambda}-1)(\sqrt{\lambda}+1)^{4}} \\
& -\frac{2 \lambda^{1-\frac{3 d}{2}}\left(2 \lambda^{\frac{d}{2}+1}+3 \lambda^{\frac{d}{2}+2}+\lambda^{\frac{d}{2}+3}+\lambda^{\frac{d+1}{2}}+3 \lambda^{\frac{d+3}{2}}+2 \lambda^{\frac{d+5}{2}}-\lambda^{3 / 2}-3 \lambda^{5 / 2}-\lambda^{7 / 2}-2 \lambda^{3}-2 \lambda^{2}\right)}{a^{2}(\sqrt{\lambda}+1)^{4}\left(\lambda^{3 / 2}+\lambda^{5 / 2}-\lambda-1\right)} \\
& +\frac{2\left(-2 \lambda^{2-\frac{d}{2}}-2 \lambda^{3-\frac{d}{2}}-\lambda^{\frac{3}{2}-\frac{d}{2}}-3 \lambda^{\frac{5}{2}-\frac{d}{2}}-\lambda^{\frac{7}{2}-\frac{d}{2}}+5 \lambda^{3 / 2}+3 \lambda^{5 / 2}+\lambda^{3}+5 \lambda^{2}+3 \lambda+\sqrt{\lambda}\right)}{a(\sqrt{\lambda}+1)^{4}\left(\lambda^{3 / 2}-1\right)} \\
& -2 \sum_{p=2}^{d} \frac{a_{p} \lambda^{2}}{a^{2}\left(\lambda^{2}-\lambda_{p}\right)} \frac{\lambda^{-3 d / 2}\left(\lambda^{\frac{3}{2}}-1\right) \lambda_{p}^{d+1}}{(\sqrt{\lambda}+1)^{3}\left(\lambda^{\frac{3}{2}}-\lambda_{p}\right)} \\
& +2 \sum_{p=2}^{d} \frac{a_{p} \lambda^{2}}{a^{2}\left(\lambda^{2}-\lambda_{p}\right)}\left[\lambda ^ { - 1 - 2 d } \left(\lambda^{3} \lambda_{p}\left(\sqrt{\lambda} \sqrt{\lambda_{p}}\right)^{2 d-1}-\lambda \lambda_{p}^{3 / 2}\left(\sqrt{\lambda} \sqrt{\lambda_{p}}\right)^{2 d}-2 \lambda^{\frac{d}{2}+3} \lambda_{p}^{d+\frac{1}{2}}-2 \lambda^{\frac{d}{2}+4} \lambda_{p}^{d+\frac{1}{2}}-\lambda^{d+2} \lambda_{p}^{d+\frac{1}{2}}\right.\right. \\
& +2 \lambda^{d+\frac{5}{2}} \lambda_{p}^{d+\frac{1}{2}}+2 \lambda^{d+3} \lambda_{p}^{d+\frac{1}{2}}+2 \lambda^{d+\frac{7}{2}} \lambda_{p}^{d+\frac{1}{2}}-2 \lambda^{\frac{d+7}{2}} \lambda_{p}^{d+\frac{1}{2}}+2 \lambda^{\frac{d}{2}+2} \lambda_{p}^{d+\frac{3}{2}}-\lambda^{d+\frac{1}{2}} \lambda_{p}^{d+\frac{3}{2}}-2 \lambda^{d+1} \lambda_{p}^{d+\frac{3}{2}}-2 \lambda^{d+\frac{3}{2}} \lambda_{p}^{d+\frac{3}{2}} \\
& \left.\left.+2 \lambda^{\frac{d+3}{2}} \lambda_{p}^{d+\frac{3}{2}}+2 \lambda^{\frac{d+5}{2}} \lambda_{p}^{d+\frac{3}{2}}\right) /\left((\sqrt{\lambda}+1)^{3} \sqrt{\lambda_{p}}\left(\lambda^{3 / 2}-\lambda_{p}\right)\right)\right] .
\end{aligned}
$$


Substituting Eqs. (22), (23), (24), (25), (26), (27), (28), and (29) in Eq. (21) we obtain,

$$
\begin{aligned}
& \sigma^{(1)}(z)=\frac{a^{2} \lambda^{-2 d}}{2}\left[2 d-1+\frac{2 \sqrt{\lambda}}{\lambda-1}+\frac{\sqrt{\lambda}+\lambda+\lambda^{3 / 2}}{(\sqrt{\lambda}-1)(\sqrt{\lambda}+1)^{3}}+\frac{\lambda^{2}}{a^{2}(\sqrt{\lambda}-1)(\sqrt{\lambda}+1)^{3}}-2 \sum_{p=2}^{d} \frac{a_{p}\left(\lambda^{2}-\lambda_{p}\right)}{a\left(1-\frac{\lambda_{p}}{\lambda}\right)(\sqrt{\lambda}+1)\left(\lambda^{3 / 2}-\lambda_{p}\right)}\right. \\
& -\sum_{p, q=2}^{d} \frac{a_{p} a_{q}(\sqrt{\lambda}-1)\left(\lambda^{3}-\lambda_{p} \lambda_{q}\right)}{a^{2}\left(1-\frac{\lambda_{p} \lambda_{q}}{\lambda^{2}}\right)(\sqrt{\lambda}+1)\left(\lambda^{3 / 2}-\lambda_{p}\right)\left(\lambda^{3 / 2}-\lambda_{q}\right)}-2 \sum_{p=2}^{d} \frac{a_{p}\left(2 \lambda^{2}+2 \lambda^{5 / 2}+\lambda^{3}-\lambda_{p}(1+2 \sqrt{\lambda}+2 \lambda)\right)}{a\left(1-\frac{\lambda_{p}}{\lambda}\right)(\sqrt{\lambda}+1)^{3}\left(\lambda^{3 / 2}-\lambda_{p}\right)} \\
& -\sum_{p, q=2}^{d} \frac{\left.a_{p} a_{q}\left(\lambda^{3}\left(\lambda^{3 / 2}+\lambda+\sqrt{\lambda}-1\right)-\lambda^{2}\left(\lambda_{p}+\lambda_{q}\right)(1+\sqrt{\lambda})+\lambda_{p} \lambda_{q}\left(1+\sqrt{\lambda}+\lambda-\lambda^{3 / 2}\right)\right)\right)}{a^{2}\left(1-\frac{\lambda_{p} \lambda_{q}}{\lambda^{2}}\right)(\sqrt{\lambda}+1)^{3}\left(\lambda^{3 / 2}-\lambda_{p}\right)\left(\lambda^{3 / 2}-\lambda_{q}\right)} \\
& -\frac{2 \lambda^{(1-3 d) / 2}\left(a(1-3 d) \lambda^{d}-\frac{\lambda}{\lambda-1}\right)}{a^{2}(\sqrt{\lambda}+1)}-\frac{2 \lambda^{(1-d) / 2}(1+3 d(\sqrt{\lambda}-1))}{a(\lambda-1)}-\frac{2 \lambda^{-3(d-1) / 2}}{a^{2}(\sqrt{\lambda}+1)^{2}\left(\lambda^{3 / 2}-1\right)} \\
& -2 \sum_{p=2}^{d} \frac{a_{p} \lambda^{-3(d-1) / 2} \lambda_{p}^{d}}{a^{2}\left(\lambda-\lambda_{p}\right)(\sqrt{\lambda}+1)}\left(1-\frac{(\sqrt{\lambda}-1) \lambda_{p}}{\lambda^{3 / 2}-\lambda_{p}}\right)-\frac{2 \lambda^{(1-2 d) / 2}(1+\sqrt{\lambda}+\lambda)\left(a(1-2 d) \lambda^{d}-\frac{\lambda}{\lambda-1}\right)}{a^{2}(\sqrt{\lambda}+1)^{3}} \\
& -\frac{2 \lambda^{(3-2 d) / 2}}{a^{2}(\sqrt{\lambda}-1)(\sqrt{\lambda}+1)^{4}}-\frac{2 \sqrt{\lambda}(1+\sqrt{\lambda}+\lambda)(1+2 d(\sqrt{\lambda}-1))}{a(\sqrt{\lambda}+1)^{3}(\sqrt{\lambda}-1)} \\
& -2 \sum_{p=2}^{d} \frac{a_{p} \lambda^{(3-2 d) / 2} \lambda_{p}^{d}(1+\sqrt{\lambda}+\lambda)}{a^{2}\left(\lambda-\lambda_{p}\right)(\sqrt{\lambda}+1)^{3}}\left(1-\frac{(\sqrt{\lambda}-1) \lambda_{p}}{\lambda^{3 / 2}-\lambda_{p}}\right)-\frac{2 \lambda^{2-\frac{3 d}{2}}}{a^{2}(\sqrt{\lambda}+1)^{3}\left(\lambda^{2}-1\right)}+\frac{2(\lambda+\sqrt{\lambda}+1) \lambda^{1-\frac{d}{2}}}{a(\sqrt{\lambda}-1)(\sqrt{\lambda}+1)^{4}} \\
& -\frac{2\left(-2 \lambda^{2-\frac{d}{2}}-2 \lambda^{3-\frac{d}{2}}-\lambda^{\frac{3}{2}-\frac{d}{2}}-3 \lambda^{\frac{5}{2}-\frac{d}{2}}-\lambda^{\frac{7}{2}-\frac{d}{2}}+5 \lambda^{3 / 2}+3 \lambda^{5 / 2}+\lambda^{3}+5 \lambda^{2}+3 \lambda+\sqrt{\lambda}\right)}{a(\sqrt{\lambda}+1)^{4}\left(\lambda^{3 / 2}-1\right)} \\
& +\frac{2 \lambda^{1-\frac{3 d}{2}}\left(2 \lambda^{\frac{d}{2}+1}+3 \lambda^{\frac{d}{2}+2}+\lambda^{\frac{d}{2}+3}+\lambda^{\frac{d+1}{2}}+3 \lambda^{\frac{d+3}{2}}+2 \lambda^{\frac{d+5}{2}}-\lambda^{3 / 2}-3 \lambda^{5 / 2}-\lambda^{7 / 2}-2 \lambda^{3}-2 \lambda^{2}\right)}{a^{2}(\sqrt{\lambda}+1)^{4}\left(\lambda^{3 / 2}+\lambda^{5 / 2}-\lambda-1\right)} \\
& -2 \sum_{p=2}^{d} \frac{a_{p} \lambda^{2}}{a^{2}\left(\lambda^{2}-\lambda_{p}\right)}\left[\lambda ^ { - 1 - 2 d } \left(\lambda^{3} \lambda_{p}\left(\sqrt{\lambda} \sqrt{\lambda_{p}}\right)^{2 d-1}-\lambda \lambda_{p}^{3 / 2}\left(\sqrt{\lambda} \sqrt{\lambda_{p}}\right)^{2 d}-2 \lambda^{\frac{d}{2}+3} \lambda_{p}^{d+\frac{1}{2}}-2 \lambda^{\frac{d}{2}+4} \lambda_{p}^{d+\frac{1}{2}}-\lambda^{d+2} \lambda_{p}^{d+\frac{1}{2}}\right.\right. \\
& +2 \lambda^{d+\frac{5}{2}} \lambda_{p}^{d+\frac{1}{2}}+2 \lambda^{d+3} \lambda_{p}^{d+\frac{1}{2}}+2 \lambda^{d+\frac{7}{2}} \lambda_{p}^{d+\frac{1}{2}}-2 \lambda^{\frac{d+7}{2}} \lambda_{p}^{d+\frac{1}{2}}+2 \lambda^{\frac{d}{2}+2} \lambda_{p}^{d+\frac{3}{2}}-\lambda^{d+\frac{1}{2}} \lambda_{p}^{d+\frac{3}{2}}-2 \lambda^{d+1} \lambda_{p}^{d+\frac{3}{2}}-2 \lambda^{d+\frac{3}{2}} \lambda_{p}^{d+\frac{3}{2}} \\
& \left.\left.\left.+2 \lambda^{\frac{d+3}{2}} \lambda_{p}^{d+\frac{3}{2}}+2 \lambda^{\frac{d+5}{2}} \lambda_{p}^{d+\frac{3}{2}}\right) /\left((\sqrt{\lambda}+1)^{3} \sqrt{\lambda_{p}}\left(\lambda^{3 / 2}-\lambda_{p}\right)\right)\right]+2 \sum_{p=2}^{d} \frac{a_{p} \lambda^{2}}{a^{2}\left(\lambda^{2}-\lambda_{p}\right)} \frac{\lambda^{-3 d / 2}\left(\lambda^{\frac{3}{2}}-1\right) \lambda_{p}^{d+1}}{(\sqrt{\lambda}+1)^{3}\left(\lambda^{\frac{3}{2}}-\lambda_{p}\right)}\right] .
\end{aligned}
$$

The variation interfacial tension, $\sigma(z)=\sigma^{(0)}(z)+z_{D} \sigma^{(1)}(z)$, with $z$ is shown in Fig. 2 The function decreases monotonically with decreasing $z$ till it becomes zero at $z_{c}$. Near $z_{c}$, it varies as $\left|z-z_{c}\right|$.

\section{CALCUlation OF $\rho(d)$ WITH ONE DEFECT}

The bulk density may be calculated from $\Omega_{e e}$ (Eq. (15)),

$$
\Omega_{e e}=\left[\omega_{0}(N)\right]^{M / 2}\left(1+z_{D} \frac{M}{2} \frac{a^{2}}{\lambda^{2 d}}[(N-d+1)+2 f(0)]+O\left(z_{D}^{2}\right)\right) .
$$

At thermodynamic limit, free energy per site is thus given by,

$$
f=-\frac{1}{2}\left[\ln \lambda+z_{D} a^{2} \lambda^{-2 d}\right]+O\left(z_{D}^{2}\right) .
$$

Density of rectangles, $\rho(d)$ is defined as

$$
\rho(d)=-d z \frac{d}{d z} f,
$$




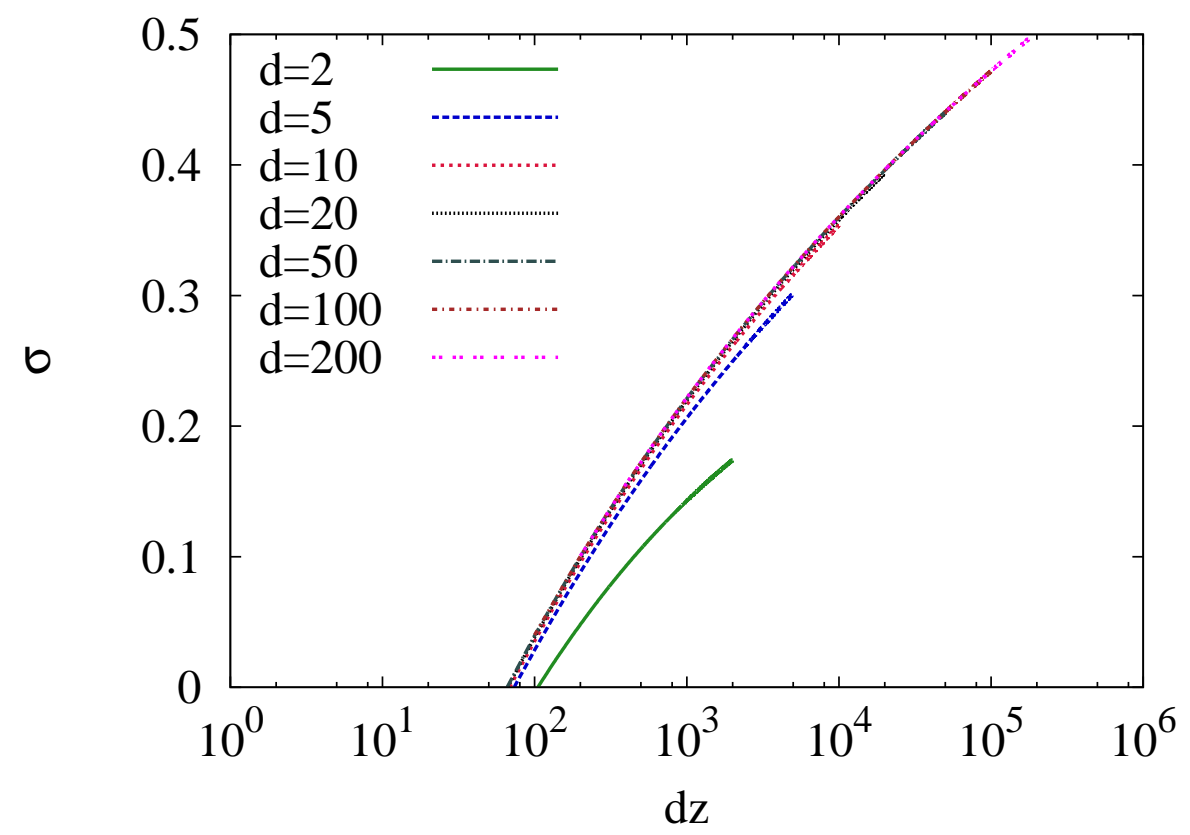

FIG. 2. Variation of interfacial tension $\sigma(z)$ truncated at order $z_{D}$ with $d z$

which may be simplified to

$$
\rho(d)=1-\frac{a}{\lambda}+z_{D} a^{2} \lambda^{-1-2 d}[2 a(d(a+1)-1)-\lambda(d(2 a+1)-2)]+O\left(z_{D}^{2}\right) .
$$

\section{CALCULATION OF CONTRIBUTION FROM OVERHANG}

A configuration without any overhangs correspond to a PDSAW with only left, right and down steps allowed. We denote weight for vertical downward step as $\mathbb{D}$, upward step as $\mathbb{U}$, left step as $\mathbb{L}$ and right step as $\mathbb{R}$. The steps will be denoted by $D, U, L$ and $R$ respectively, where

$$
\begin{array}{r}
\mathbb{D}=a z \lambda^{-d}, \\
\mathbb{R}=\mathbb{L}=\lambda^{-1 / 2} .
\end{array}
$$

A PDSAW consists of an arbitrary concatenation of substrings $D, D R, D R^{2}, \ldots, D L, D L^{2}, \ldots$ etc. Hence the PDSAW has a formal generating function

$$
\wp=\frac{1}{1-D-D R^{*}-D L^{*}},
$$

where

$$
R^{*}=R+R^{2}+R^{3}+\ldots=\frac{R}{1-R} .
$$

and a similar equation for $L^{*}$. Correspondingly, the equation for the evaluation of this generating function is

$$
W=\frac{1}{1-\mathbb{D}-\mathbb{D R}^{*}-\mathbb{D L}^{*}} .
$$

The criticality condition when the generating function diverges, then becomes

$$
\mathbb{D}\left(1+\mathbb{R}^{*}+\mathbb{L}^{*}\right)=1 .
$$


which is the condition of interfacial tension $\sigma^{(0)}(z)$ becoming zero. Using Eq. (10) of main text, this may be simplified to give

$$
a(1+1 / \sqrt{\lambda})^{2}=1
$$

Now consider interface configurations with overhangs of height 1 . The simplest sequence of steps corresponding to an overhang is $D R^{a} U R^{b}$, with $a, b \geq d$. Let us define

$$
W_{R}=\sum_{a, b=d}^{\infty} R^{a} U R^{b} D
$$

and arbitrary concatenation of such overhangs at same height is given by

$$
W_{R}^{*}=W_{R}+W_{R}^{2}+W_{R}^{3}+\ldots=W_{R} /\left[1-W_{R}\right]
$$

We define $W_{L}$, and $W_{L}^{*}$ similarly, with $R$ replaced by $L$, corresponding to overhangs to the left of the interface. Given an overhang configuration with a given values of $a$ and $b$, it is easy to see that corresponding sum over allowed rectangle configurations to the left and right of interface gives a relative weight $z^{2} \omega(a-d) \omega(b-d) \lambda^{-3(a+b) / 2}$. Then summing over $a$, and $b$, we get

$$
\mathbb{W}_{R}=\sum_{a=d}^{\infty} \sum_{b=d}^{\infty} z^{2} \lambda^{-\frac{3}{2}(a+b)} \omega_{0}(a-d) \omega_{0}(b-d),
$$

which, using Eq. (12) of main text can be simplified to

$$
\begin{aligned}
\mathbb{W}_{R} & =\left[z \lambda^{-\frac{3 d}{2}}\right]^{2}\left[\sum_{n=0}^{\infty} \omega_{0}(n) \lambda^{-\frac{3 n}{2}}\right]^{2} \\
& =\left[\frac{z \lambda^{-\frac{3 d}{2}}}{1-\lambda^{-3 / 2}-\left(1-\lambda^{-1}\right) \lambda^{-d / 2}}\right]^{2}
\end{aligned}
$$

A general configuration with overhangs of height at most 1 is an arbitrary concatenation of subsequences $D, D R^{*}, D L^{*}, X_{L}$ and $X_{R}$, where

$$
\begin{aligned}
& X_{R}=D\left(\left(\sum_{n \geq 0} L^{n} D R^{n}\right)+R^{*} D\right) W_{R}^{*}\left(1+R^{*}\right) \\
& X_{L}=D\left(\left(\sum_{n \geq 0} R^{n} D L^{n}\right)+L^{*} D\right) W_{L}^{*}\left(1+L^{*}\right) .
\end{aligned}
$$

Thus the criterion for divergence of the generating function Eq. (40) gets modified to

$$
\mathbb{D}\left(1+2 \mathbb{R}^{*}+2\left(\frac{1}{1-\mathbb{L} \mathbb{R}}+\mathbb{R}^{*}\right) \frac{\mathbb{D W}_{R}\left(1+\mathbb{R}^{*}\right)}{\left(1-\mathbb{W}_{R}\right)}\right)=1
$$

which can be simplified to

$$
\frac{\lambda-1}{d(\lambda-1)+1}\left(\frac{\sqrt{\lambda}+1}{\sqrt{\lambda}-1}+\frac{2 \sqrt{\lambda}(1+\sqrt{\lambda}+\lambda) \mathbb{W}_{R}}{(d(\lambda-1)+1)(\sqrt{\lambda}-1)\left(1-\mathbb{W}_{R}\right)}\right)=1
$$

